\title{
Biomacromolecule-Assisted Synthesis and Electrocapacitive Behavior of Manganese Ferrite Nanoparticles
}

\author{
Shuibo Liu ${ }^{l}$, Zichao Li ${ }^{l}$, Xiao-xiong Wang ${ }^{2}$, Xuehua Liu ${ }^{l}$, Wanneng Ye $e^{1, *}$, Yun-ze Long ${ }^{2}$, \\ Hongliang Lil, Peizhi Guo ${ }^{l, *}$, X.S. Zhao ${ }^{l}$ \\ ${ }^{1}$ Institute of Materials for Energy and Environment, State Key Laboratory Breeding Based of New \\ Fiber Materials and Modern Textile, School of Materials Science and Engineering, Qingdao \\ University, Qingdao, 266071, P. R. China \\ ${ }^{2}$ College of Physics, Qingdao University, Qingdao 266071, P. R. China \\ *E-mail: ywn@qdu.edu.cn, pzguo@qdu.edu.cn, qduguo@163.com
}

doi: $10.20964 / 2017.12 .09$

Received: 4 June 2017 / Accepted: 28 September 2017 / Published: 12 November 2017

Two types of manganese ferrite nanoparticles, namely MF-SA and MF-CHI, have been synthesized solvothermally from the synthesis systems containing sodium alginate and chitosan, respectively. Both MF-SA and MF-CHI showed well crystalline nature based on the XRD and TEM measuremants. The larger saturation magnetization value of MF-SA should be ascribed to its slightly larger crystallite size than that of MF-CHI. Results from $\mathrm{N}_{2}$ adsorption-desorption isotherms and Raman spectra confirmed the structural difference in MF-SA and MF-CHI. The electrocapacitive behavior of manganese ferritebased supercapacitors has been studied and MF-SA displayed a specific capacitance of around 60.4 $\mathrm{F} \cdot \mathrm{g}^{-1}$ at $0.25 \mathrm{~A} \bullet \mathrm{g}^{-1}$ in aqueous symmetric supercapacitors, larger than that of MF-CHI about $45.1 \mathrm{~F} \cdot \mathrm{g}^{-1}$ at the same condition. MF-SA based supercapacitor also showed better capacitance maintenance of

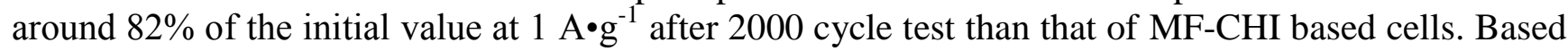
on the experimental data, the structure-property relationship of two types of $\mathrm{MnFe}_{2} \mathrm{O}_{4}$ nanoparticles have been discussed and analyzed.

Keywords: Ferrite, Nanoparticle, Marine biomolecule, Roman spectroscopy, Supercapacitor

\section{$\underline{\text { FULL TEXT }}$}

(C) 2017 The Authors. Published by ESG (www.electrochemsci.org). This article is an open access article distributed under the terms and conditions of the Creative Commons Attribution license (http://creativecommons.org/licenses/by/4.0/). 